\title{
Towards Swarm Calculus: Universal Properties of Swarm Performance and Collective Decisions
}

\author{
Heiko Hamann \\ Artificial Life Laboratory of the Department of Zoology \\ Karl-Franzens University Graz, Graz, Austria, \\ heiko.hamann@uni-graz.at
}

July 26, 2012

\begin{abstract}
The search for generally applicable methods in swarm intelligence aims to gain new insights about natural swarms and to develop design methodologies for artificial swarms. The ideal would be a 'swarm calculus' that allows to calculate key features such as expected swarm performance and robustness on the basis of a few parameters. A path towards this ideal is to find methods and models that have maximal generality. We report two models that might be examples of exceptional generality. First, we present an abstract model that describes the performance of a swarm depending on the swarm density based on the dichotomy between cooperation and interference. Second, we give an abstract model for decision making that is inspired by urn models. A parameter, that controls the feedback based on the current consensus, allows to understand the effects of an increasing probability for positive feedback over time in a decision making system.
\end{abstract}

\section{Introduction}

Research in the context of swarm intelligence is important in biology to gain new insights about natural swarms and also in fields aiming for artificial swarms, such as swarm robotics, to obtain sophisticated design methodologies. The ideal tools would allow to calculate swarm behavior, performance, stability, and robustness based on few observed parameters in case of a natural swarm system or based on few designed parameters in case of an artificial swarm. We call this highly desired set of tools 'swarm calculus' (calculus in its general sense).

Models will surely be an important part of swarm calculus. In order to define a general methodology of understanding and designing swarm systems, general properties and generally applicable models need to be found. Today only few models exist that have the potential to become general swarm models. Biological swarm models are particularly distinguished by their variety Okubo and Levin, 2001, Okubo, 1986, Vicsek and Zafeiris, 2012, Edelstein-Keshet, 2006, Camazine et al., 
2001]. Typically each biological challenge is answered by a specialized model. The desire for a model with applicability to all natural swarms seems to be small in that community. In the field of artificial swarms, such as robot swarms, the desire for generality seems to be bigger which is, for example, expressed by several models of swarm robotics Hamann, 2010, Berman et al., 2011, Prorok et al., 2011, Milutinovic and Lima, 2007]. The idea of these models is to support the design of swarm robotic systems within a maximal range of applications. They focus on features that describe quantities of the swarm behavior, such as robot distributions or required times for certain tasks and typically struggle between the intended generality and having direct connections between the model and the robot behavior. If we abandon, however, the demand for a detailed description of behavioral features and focus only on high-level features such as overall performance or the macroscopic process of a collective decision then a higher degree of generality is achievable. In this paper, we identify two models of universal properties of swarm systems concerning the dependence of swarm performance on swarm density and the dependence of collective decisions on positive feedback.

\section{Universal Properties of Swarm Performance}

It is quite clear that a function of swarm performance depending on swarm density cannot be a simple linear function. For a true swarm scenario a very low density (e.g., corresponding to one agent in the whole area) has to result in low performance because there is no cooperation. With increasing density the performance increases because more and more cooperation is possible (assuming that cooperation is an essential beneficial part of swarms). Even a superlinear performance increase is possible in this interval Mondada et al., 2005]. At some critical/optimal density the improvement in cooperation possibilities will be lower than the drawback of high densities, namely interference Lerman and Galstyan, 2002]. With further increase of the density the performance is decreasing. Hence, swarms generally face a tradeoff between beneficial cooperation and obstructive interference.

It turns out that not only these qualitative properties are similar in many swarm systems but also the actual shapes of swarm performance over swarm size plots (see Fig. 1(a) . Examples are the performance of foraging in a group of robots (Fig. 1(b) and Fig. 10a in Lerman and Galstyan, 2002]), the activation dynamics and information capacity in an abstract cellular automaton model of ants (Figs. 1b and 1c in Miramontes, 1995]), and even in the sizes of social networks (Fig. 8b in Strogatz, 2001]). Furthermore, notice that these shapes are typical in probability distributions such as Weibull, Wigner, and log-normal. A related set of models are traffic models of flow over density. The 'fundamental diagram' of traffic flow [ighthill and Whitham, 1955] is symmetric, more realistic models propose at least two asymmetric phases of free and synchronized flow. Actual measurements on highways show curves with shapes similar to Fig. 1(a) (e.g., see Fig. 6-4 in Mahmassani et al., 2009]). In these models, there exist two den- 
sities for a given flow (except for the maximum flow) similar to the situation here where we have two swarm densities for a given swarm performance.

Having identified the two main components (cooperation and interference) and the typical shape of these plots we can define a simple model. The idea is to fit this model to empirical data for verification and predictions.

\subsection{Simple model of swarm performance}

For a given bounded, constant area $A$ the swarm density $\rho$ is defined by the swarm size $N$ according to $\rho=N / A$. We define the swarm performance $P$ depending on swarm size $N$ by

$$
P(N)=C(N)(I(N)-d)=a_{1} N^{b} a_{2} \exp (c N),
$$

for parameters $c<0, a_{1}, a_{2}, b>0$, and $d \geq 0$ (see Fig. 1(a) . Parameter $d$ is subtracted to force a decrease to zero $\left(\lim _{N \rightarrow \infty} I(N)-d=0\right)$. The swarm performance depends on two components $C$ and $I$. First, the swarm effort without negative feedback is defined by the cooperation function (see also Fig. 1(a)

$$
C(N)=a_{1} N^{b} .
$$

This function can be interpreted as the potential for cooperation in a swarm that would exist without certain constraints, such as physical collisions. The same formula was used by Breder [Breder, 1954] to model the cohesiveness of a fish school and by Bjerknes and Winfield Bjerknes and Winfield, 2010] to model swarm velocity in emergent taxis. However, they used parameters of $b<1$ while we are using mostly values of $b>1$. Second, the interference function (see also Fig. 1(a) is defined by

$$
I(N)=a_{2} \exp (c N)+d
$$

with $d$ used for scaling (e.g., $\lim _{N \rightarrow \infty} I(N)=d$ ). The exponential decrease

seems to be a reasonable choice, for example, compare Fig. 10b in Lerman and Galstyan, 2002] which shows an exponentially decreasing efficiency per robot in a foraging task.

\section{$2.2 \quad$ Examples}

To prove the wide applicability of this simple model we fit it to some swarm performance plots that were available. We briefly investigate four scenarios: foraging in a group of robots Lerman and Galstyan, 2002|, collective decision making [Hamann et al., 2012] based on BEECLUST [Schmickl and Hamann, 2011], aggregations in tree-like structures and reduction to shortest paths Hamann, 2006] similar to Hamann and Wörn, 2008], and the emergent taxis scenario (also sometimes called 'alpha algorithm') [Nembrini et al., 2002, Bierknes et al., 2007].

Given the data of the the overall performance, the four parameters of eq. 1 can be directly fitted to it. That is what we do for the first three of our four 
examples in Fig. 1. The equation can be well fitted to the empirical data. In case of the foraging scenario (Fig. 1(b) we also have data about the efficiency per robot. We can use the model parameters, that were obtained by fitting the model to the overall performance, to predict the efficiency per robot. This is done by scaling the interference function linearly and plotting it against the efficiency per robot. The satisfying result is shown in Fig. 1(b).

We analyze the forth example, emergent taxis, in more detail. The following empirical data is based on a simple simulation. This simulation is noise-free and therefore robots move in straight lines except for $\mathrm{u}$-turns according to the emergent taxis algorithm. First, we measure the performance that is achieved without cooperation. This is done by defining a random behavior that ignores any characteristic feature of the actual emergent taxis algorithm. For example, in the emergent taxis algorithm, robots count the number of neighbors and do $\mathrm{u}$-turns if this number drops below a threshold $\alpha$. To obtain the cooperationfree behavior we have set this parameter to $\alpha=0$ in the simulation. Hence, no robot will ever u-turn and they basically disperse in the arena. A simulation run is stopped once a robot touches a wall. The performance of the swarm $P$ is measured by the total distance covered by the swarm's barycenter multiplied by the swarm size (i.e., an estimate of how much distance was effectively covered summed over all robots). The performance obtained by this random behavior can be fitted using the interference function of eq. 3. The well fitted interference function and the empirically obtained data is shown in Fig. 2(a) labeled 'random'. In a second step, we keep the interference function fixed and fit the full model of swarm performance $P$ (eq. 1) to the data from the actual emergent taxis scenario by only varying the cooperation function (i.e., fitting $a_{1}$ and $b$ while keeping $a_{2}, c$ and $d$ fixed). The fitted swarm performance mode 1 is shown in Fig. 2(a) labeled 'emergent taxis'. This simple model is capable of predictions, if the interference function has been fitted and we fit the cooperation function only to a small interval of, for example, $N \in[15,25]$ (i.e., including the maximum performance).

Note that we are working with a single value (average) to describe the performance which does not fully catch the system's behavior. At least in some scenarios, as here in the emergent taxis scenario, the performance does not just decrease due to increasing interference. Instead, two coexisting phases of behaviors emerge: functioning swarms moving forward and pinned swarms with extreme numbers of u-turns. In emergent taxis this is shown, for example, by a histogram of barycenter speeds in Fig. 2(b). For $N<15$ the mean of a unimodal distribution increases with increasing $N$. Starting at about $N=15$ a second phase of slowly moving swarms emerges generating a bimodal distribution. Hence, given the fully deterministic implementation of our simulation, there are two classes of initial states (robot positions and orientations) that determine the two extremes of success or total failure. In other scenarios the interference might be increased in a different process continuously, for example, by saturation of target areas with robots.

\footnotetext{
${ }^{1}$ Fitted parameters: $a_{1}=0.01061, b=3.237, a_{2}=0.2138, c=-0.1823, d=0.075$
} 


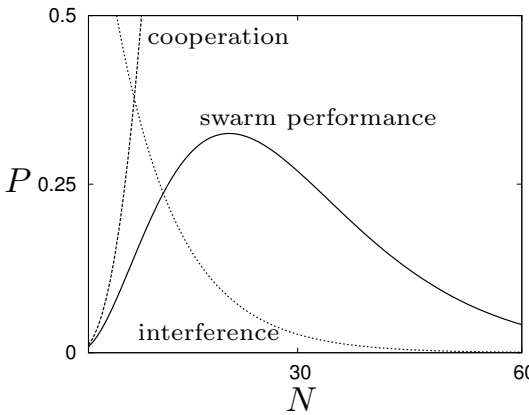

(a) Model of cooperation and interference, examples of swarm performance (eq.1), cooperation (eq. 2), and interference (eq. 3) depending on swarm size.

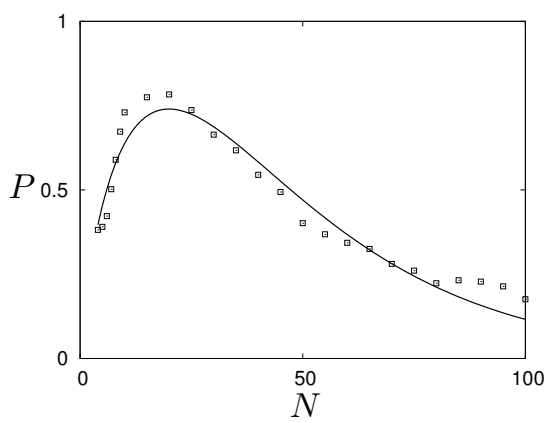

(c) Collective decision making Hamann et al., 2012 based on BEECLUST Schmickl and Hamann, 2011]; values of $P=1$ would indicate that $100 \%$ of the swarm have found a decision; $P=0$ indicates symmetry between options

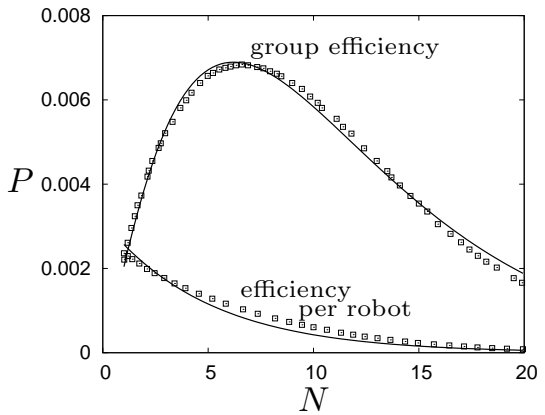

(b) Foraging in a group of robots, eq. 1 fitted to group efficiency (upper solid line), prediction of interference (lower solid line, efficiency per robot, linearly rescaled), data points extracted from Fig. 10 in Lerman and Galstyan 2002]; $P$ gives group/robot efficiency

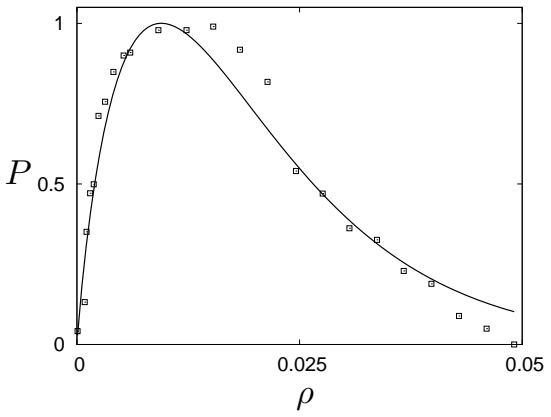

(d) Aggregation in tree-like structures and reduction to shortest path Hamann, 2006]; $P$ gives the ratio of successful runs

Figure 1: Model of cooperation and interference and three scenarios with fitted performance $P$ according to eq. 1 


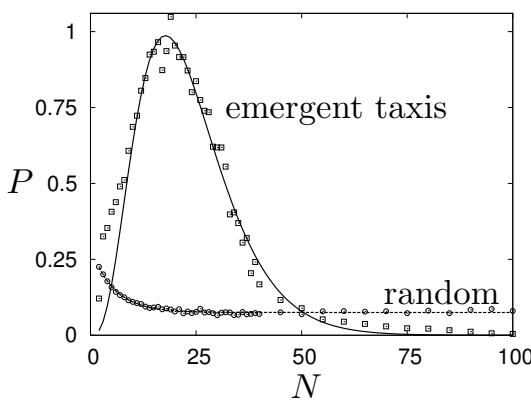

(a) Model fitted to data of random and emergent-taxis behavior; $P$ is covered distance by barycenter times $N$.

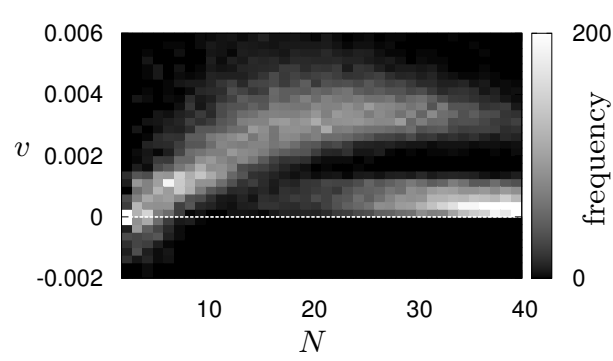

(b) Histogram of barycenter speeds $v$ for different swarm sizes. Note the bimodality about in the interval $N \in[16,40]$.

Figure 2: Performance of a random behavior and the actual self-organized emergent taxis behavior (also sometimes called 'alpha algorithm') Nembrini et al., 2002, Bjerknes et al., 2007] with fitted model (eq. 1); histogram showing two phases.

\section{Universal properties of collective decisions}

In the following we investigate macro models of collective decisions. One of the most general and at the same time simplest model of collective decisions is a model of only one state variable $s(t)$ which gives the temporal evolution of a swarm fraction that is in favor of one of the options in a binary decision process. If we assume that there is no initial bias to either option (i.e., full symmetry), then we need a tie breaker for $s=0.5$. A good choice for the tie breaker is noise because any real swarm will be noisy. The average change of $s$ depending on itself per time $(\overline{\Delta s(s) / \Delta t})$ is of interest. Given that the system should be able to converge to one of two options plus the symmetric case of $s=0.5$ we end up with at least three zeros for $\overline{\Delta s(s) / \Delta t}$ and consequently at least a cubic function. Instead of developing a model, that predefines such a function, we prefer a model that allows this function to emerge from a simple process. In swarms the tendency to a certain option once symmetry is overcome (say, for $s=0.5+\epsilon$ towards $s=1$ ) is typically a result of positive feedback. Hence, we define such a process depending on probabilities of positive feedback next.

\subsection{Simple model of collective decisions}

We use simple models inspired by the urn model of Ehrenfest and Ehrenfest 1907] which they introduced in the context of statistical mechanics and entropy. Eigen and Winkler [1993] reported similar models to show the effect of positive feedback. Here we use an urn model that has optionally positive or negative feedback depending on the system's current state and depending on a stochastic process. The urn is filled with $N$ marbles which are either red or blue. The game's dynamics is turn-based. First a marble is drawn with replacement 
followed by replacing a second one influenced by the color of the first marble. The probability of drawing a blue marble is implicitly determined by the current number of blue marbles $B(t)$ in the urn. The subsequent replacement of a second marble effects either a positive or a negative feedback. The feedback is determined explicitly by a probability

$$
P(s, \varphi)=\varphi \sin (\pi s)
$$

that is based on the current ratio $s$ of either the blue marbles in the urn $s=b(t)$ or the ratio of red marbles $s=r(t)$. The constant $\varphi \in[0,1]$ defines the 'sign' and the intensity of the feedback. For $\varphi<0.5$ negative feedback is predominant and for $\varphi>0.5$ an interval around $s=0$ emerges for which positive feedback is predominant. Say we draw a blue marble, we notice the color, and put it back into the urn. Then our model defines that with probability $P(b(t), \varphi)$ a red marble will be replaced by a blue one (i.e., a positive feedback event because drawing a blue one increased the number of blue marbles) and with probability $1-P(b(t), \varphi)$ a blue one will be replaced by a red one (i.e., a negative feedback event because now drawing a blue one decreased the number of blue marbles). Hence, the probability $P(s, \varphi)$ gives the probability of positive feedback. $P(s, \varphi)$ is plotted for different settings of $\varphi$ in Fig. 3(a) There is maximum probability for positive feedback for the fully symmetric case of $s=0.5$ as clearly seen in Fig. $3(\mathrm{a})$. For $s=0$ and $s=1$ we have $P(s, \varphi)=0$ because no positive feedback is possible (either all marbles are already blue or all marbles are red and therefore no blue one can be drawn). For $\varphi \leq 0.5$ the probability of positive feedback is small $(\varphi \leq 0.5, \forall s: P(s, \varphi) \leq 0.5)$, consequently the system is stable and kept around $s=0.5$. The analogy of this model to a collective decision making scenario is the following. The initial drawing resembles the frequency of individual decisions in the swarm proportional to $s$ within the turn-based model. The replacement of the second marble resembles the effect of a swarm member convincing another one about its decision or of being convinced of the opposite. Based on the above definitions the average expected change $\overline{\Delta B}$ of blue marbles $B$ can be calculated by summing over the four cases: drawing a blue or red marble, followed by positive or negative feedback, multiplied by the 'payoff' in terms of blue marbles respectively. Using the symmetry $P(b, \varphi)=P(1-b, \varphi)$ we get

$$
\begin{aligned}
\Delta B(b)= & b P(b, \varphi)(+1)+b(1-P(b, \varphi))(-1) \\
& +(1-b) P(1-b, \varphi)(-1)+(1-b)(1-P(1-b, \varphi))(+1) \\
= & 4(P(b, \varphi)-0.5)(b-0.5) .
\end{aligned}
$$

In Fig. 3(b) we compare the theoretical average change per round $\overline{\Delta B / \Delta t}$ according to eq. 5 to the empirically obtained average change of $B(t)$ in terms of number of marbles for the different settings of $\varphi$. The agreement between theory and empiric data is close to perfect as expected. Two zeros $s_{1}$ and $s_{2}$ emerge additionally to $s_{0}=0.5$ for $\varphi>0.5: s_{1}=\frac{1}{\pi} \arcsin \left(\frac{1}{2 \varphi}\right)$ and $s_{2}=1-\frac{1}{\pi} \arcsin \left(\frac{1}{2 \varphi}\right)$. Positive values of $\overline{\Delta B(s) / \Delta t}$ for $s<0.5$ represent dynamics that has a bias 
towards $s=0.5$ and negative values represent dynamics with a bias towards $s=0$ and vice versa for the other half $(s>0.5)$.

Fig. 3(c) gives an estimate of the asymptotic behavior of this urn model for varied feedback intensity $\varphi$. It shows a pitchfork bifurcation at $\varphi=0.5$ which is to be expected based on Fig. 3(b). Between $\varphi=0.5$ and $\varphi=0.75$ the curve defined by $\overline{\Delta B(s) / \Delta t}$ becomes cubic and generates two new stable fixed points while the former at $s=0.5$ becomes unstable.

\section{$3.2 \quad$ Examples}

Next we want to compare the data from our urn model (Fig. 3(b)) to data from more complex models, such as the density classification scenario Hamann and Wörn, 2007]. First we need a more general equation than eq. 5 . We obtain it by introducing a scaling constant $c$ that scales the average change for payoffs different from 1.

$$
\Delta s(s)=4 c(P(s, \varphi)-0.5)(s-0.5)
$$

The density classification scenario [Hamann and Wörn, 2007] is about a swarm of red and green agents moving around randomly. Their only interaction is constantly keeping track of those agents' colors they bump into. Once an agent has seen five agents of either color it changes its own color to that it has encountered most. Here, $s$ gives the ratio of red agents. The name of this scenario is due to the idea that the swarm should converge to that color that was initially superior in numbers. It turns out that the averaged change $\overline{\Delta s(s) / \Delta t}$ (see Fig. $3(\mathrm{~d})$ ) starts with a curve similar to that of $\varphi=0$ in Fig. 3(b) and then converges slowly to a curve that is similar to that of $\varphi=0.75$. Early in the simulation there is mostly negative feedback forcing values close to $s=0.5$. With increasing time the negative feedback decreases which results finally in positive feedback for $s \in[0.23,0.77]$. Comparing Fig. 3(b) to Fig. 3(d) indicates a good qualitative agreement between our urn model and the density classification scenario. Given that the curves in Fig. 3(d) converge over time to the final shape which is resembled by our model for increasing $\varphi$ in Fig. 3(b) one can say that positive feedback builds up slowly over time in the density classification scenario. By fitting eq. 6 to the data shown in Fig. 3(d) we get estimates for the feedback intensity $\varphi$. From the earliest and steepest line to the latest and only curve with positive slope in $s=0.5$ we get values of $\varphi \in[0,0,0,0.007,0.304,0.603]$ for times $t \in[100,200,400,800,1600,3200]$. By continuing this fitting for additional data not shown in Fig. 3(d)] we are able to investigate the temporal evolution of feedback intensity $\varphi$ according to our model. In Fig. $3(\mathrm{e})$, the data points of feedback intensity $\varphi$ obtained by fitting are shown and also a negative exponential function that was fitted to the data. This result supports the assumption of a negative exponential increase of positive feedback in this system as already stated in Hamann et al., 2010.

Other examples showing similarities to the $\varphi=0.75$-graph in Fig. 3(b) are Figs. 2B and 3B in Yates et al. [2009] which show the drift coefficient dependent 
on the current alignment of a swarm (average velocity). While the data obtained from experiments with locusts (Fig. 2B in Yates et al., 2009]) is too noisy, we use the data from their model (Fig. 3B in Yates et al., 2009]) to fit our model. The result is shown in Fig. 3(f) . We obtain a maximal positive feedback of $\varphi=1$.

\section{Discussion and Conclusion}

We have reported two abstract swarm models with high generality because we would like to get towards a swarm calculus. The first model describes the dependency of swarm performance on swarm density by separation into two parts: cooperation and interference. It explains the existence of an optimal or critical swarm density at which the peak performance is reached. The second model describes the dynamics of collective decision processes based on the existence and intensity of feedback. It explains how the cubic functions of decision revision emerge by an increase of positive feedback over time.

The first model is simple and somewhat obvious because the existence of optimal swarm densities is well known. However, the authors are not aware of any explicit introduction of a similar model combined with a validation by fitting the model to data from diverse swarm applications. Despite its simplicity the model has the capability to give predictions of swarm performance, especially, if the available data, to which it is fitted, includes an interval around the optimal density. That way this model might serve as a swarm calculus of swarm performance. In addition, we want to draw attention to the problem of masking special density-dependent properties by only investigating the mean performance. The example shown in Fig. 2(b) documents the existence of phases in swarm systems.

The second model is also abstract but has a higher complexity and is more conclusive because it allows for mathematical derivations. Based on our urn model for positive feedback decision processes the emerging cubic function of decision revision can be derived (see eq. 55). Here this 'cubic function' is actually trigonometric but alternatively one can choose $P(s, \varphi)=\varphi\left(1-4(s-0.5)^{2}\right)$ yielding $\Delta s(s)=2(s-0.5)-16(s-0.5)^{3}$. Hence, we generate the function of decision revision based on our urn model which allows for an interpretation of how the function emerges while, for example, in Yates et al., 2009] this function is measured in a local model. Our model of collective decisions might qualify as a part of swarm calculus because those decision revision functions seem to be a general phenomenon in swarms. An interesting result is also the negatively exponential increase of the positive feedback over time in the density classification task (see Fig. 3(e)p. Note that this increase seems to be independent from respective values of $s$. Furthermore, values close to the bounds $(s \approx 1$ or $s \approx 0)$ are not observed. An investigation of the underlying processes is beyond this paper but we want to state two ideas. First, the final saturation phase $\left(\lim _{t \rightarrow \infty} \varphi=0.8\right)$ is most likely caused by explicit noise in the simulation. The agent-agent recognition rate was set to 0.8 which keeps $P(s=0.5, \varphi)<1$. Second, the initial fast increase of $\varphi$ (after a transient which might also be caused 


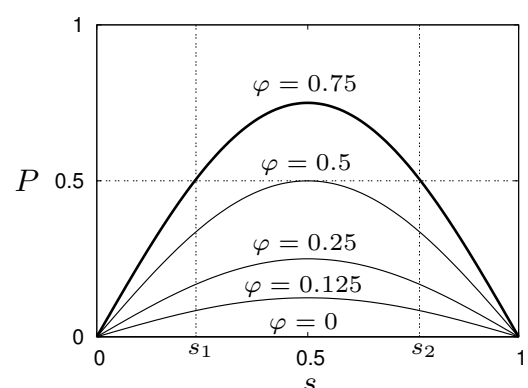

(a) Examples of setting the probability of positive feedback for intensities of feedback $\varphi \in\{0,0.125,0.25,0.5,0.75\}$.

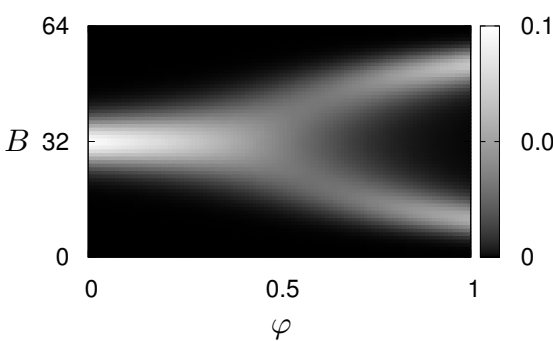

(c) Normalized histogram of blue marbles $B$ over intensity of feedback $\varphi$ after $t=200$ steps, initialized to $B(0) \in\{32,33\}$, indicating a pitchfork bifurcation at $\varphi=0.5$.

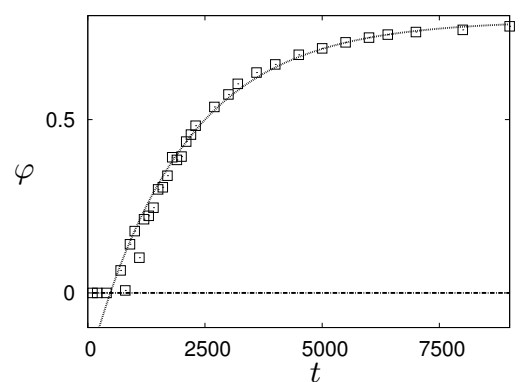

(e) Negative exponential function $\varphi(t)=$ $0.786-\exp \left(-5 \times 10^{-4} t\right)$ fitted to feedback intensities obtained from the density classification scenario.

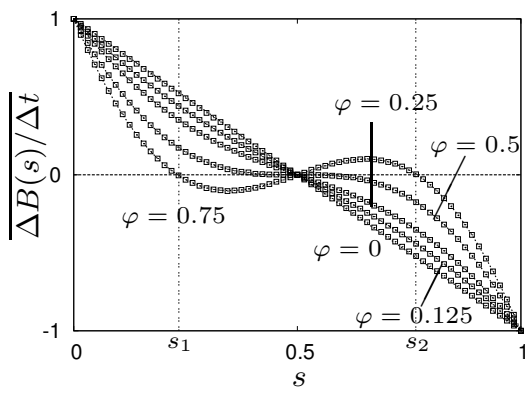

(b) Average change of $B(t)$ in terms of marbles, lines according to eq. 5 squares give empirical data, number of samples is $8 \times 10^{5}$ for each possible $s, 64$ marbles.

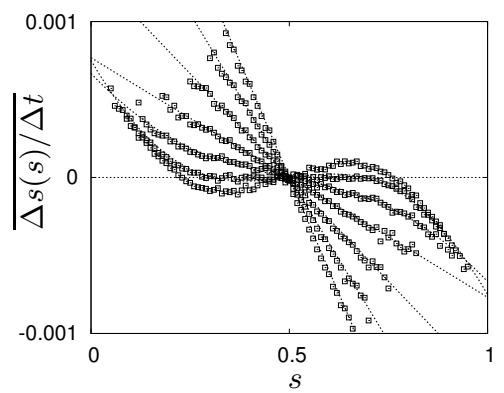

(d) Density classification scenario [Hamann and Wörn, 2007], change of the ratio of red robots for different times during simulation, squares give empirical data (from [Hamann et al., 2010]), lines are fitted according to eq. 6

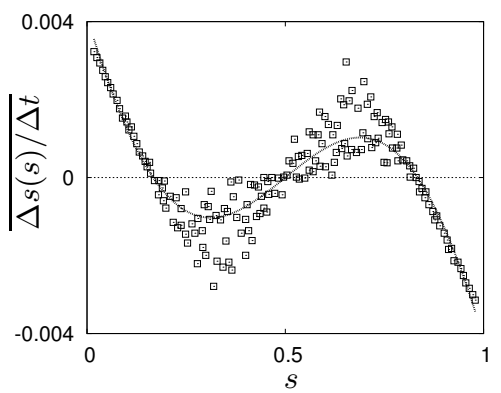

(f) Model fitted to data from Fig. 3B of Yates et al. 2009] (local model of swarm alignment in locusts) by $\varphi=1$ (and $c=$ $\left.4.134 \times 10^{-3}\right)$; data scaled to $s \in[0,1]$.

Figure 3: Settings of the positive feedback probabilities, resulting average change in $B(t)$ in the urn model over the ratio of marbles $s$, histogram of blue marbles for varied intensity of feedback $\varphi$, comparison of model and results from the density classification scenario [Hamann and Wörn, 2007], increase of positive feedback over time, and comparison of model and results from Yates et al. [2009]. 


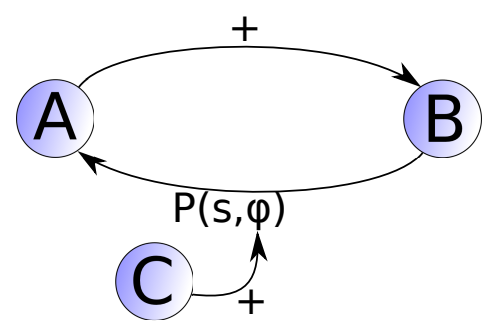

Figure 4: Time-variant feedback system; here for increasing probability of pos. feedback.

by the simulation because agents revise their color only after a minimum of five agent-agent encounters) might be caused by locally emerging sub-groups of homogeneous color within small areas that generate 'islands' of early positive feedback. Time-variant positive feedback was also observed in BEECLUSTcontrolled swarms as reported before Hamann et al., 2012]. Hence, a feedback system as given in Fig. 4 seems to be a rather common situation in swarm systems. In terms of the above urn model we can mimic this situation, say $A$ is the number of blue marbles (w.l.o.g.), $B$ is the probability of drawing a blue marble, $P$ is the probability of positive feedback (i.e., this edge can also negatively influence $A$ ), and $C$ is an unspecified state variable that increases feedback $(\varphi)$ over time and is influenced by an additional, unknown process. This triggers the question of what $C$ can be and how it influences the feedback process independent of the current swarm consensus $s$.

We get maximally positive feedback $\varphi=1$ for the data of [Yates et al., 2009] (see Fig. 3(f) with the effect that situations of low alignment $(s \approx 0.5)$ are left as fast as possible. This reinforces the findings of Yates et al. [Yates et al., 2009] about the diffusion coefficient. A major feature of the self-organizing processes in the swarm seems to be that times in states of low aligned are minimized by the system (Yates et al.: "A higher diffusion coefficient at lower alignments suggests that the locusts 'prefer' to be in a highly aligned state").

The result of this paper is that generally applicable swarm models, that have simple preconditions, exist. To apply the model of swarm performance, only a concept of swarm density is necessary and to apply the model of collective decisions only a consensus variable of a binary decision is necessary. Despite their simplicity, both models have enough explanatory power to give insights about swarm processes such as the interplay of cooperation and interference and the installation of positive feedback. Hence, we contend that it is possible to generate a set of models and methods of general applicability for swarm science, that is, to create a swarm calculus.

\section{Acknowledgments.}

The author thanks Payam Zahadat, Jürgen Stradner and the anonymous reviewers for very helpful comments that improved the manuscript. 


\section{References}

Spring Berman, Vijay Kumar, and Radhika Nagpal. Design of control policies for spatially inhomogeneous robot swarms with application to commercial pollination. In IEEE International Conference on Robotics and Automation (ICRA'11), pages 378-385, 2011.

Jan Dyre Bjerknes and Alan Winfield. On fault-tolerance and scalability of swarm robotic systems. In Proc. Distributed Autonomous Robotic Systems (DARS 2010), November 2010.

Jan Dyre Bjerknes, Alan Winfield, and Chris Melhuish. An analysis of emergent taxis in a wireless connected swarm of mobile robots. In IEEE Swarm Intelligence Symposium, pages 45-52, Los Alamitos, CA, 2007. IEEE Press.

C. M. Breder. Equations descriptive of fish schools and other animal aggregations. Ecology, 35(3):361-370, July 1954.

Scott Camazine, Jean-Louis Deneubourg, Nigel R. Franks, James Sneyd, Guy Theraulaz, and Eric Bonabeau. Self-Organizing Biological Systems. Princeton Univ. Press, 2001.

Leah Edelstein-Keshet. Mathematical models of swarming and social aggregation. Robotica, 24(3):315-324, May 2006.

Paul Ehrenfest and Tatiana Ehrenfest. Über zwei bekannte Einwände gegen das Boltzmannsche H-Theorem. Physikalische Zeitschrift, 8:311-314, 1907.

Manfred Eigen and Ruthild Winkler. Laws of the game: how the principles of nature govern chance. Princeton University Press, 1993. ISBN 9780691025667.

Heiko Hamann. Modeling and investigation of robot swarms. Master's thesis, University of Stuttgart, Germany, 2006. http://heikohamann.de/pub/hamannMasterThesis.pdf

Heiko Hamann. Space-Time Continuous Models of Swarm Robotics Systems: Supporting Global-to-Local Programming. Springer-Verlag, 2010.

Heiko Hamann and Heinz Wörn. Embodied computation. Parallel Processing Letters, 17(3):287-298, September 2007. http://heikohamann.de/pub/hamannEmbodied07.pdf

Heiko Hamann and Heinz Wörn. Aggregating robots compute: An adaptive heuristic for the Euclidean Steiner tree problem. In The tenth International Conference on Simulation of Adaptive Behavior (SAB'08), volume 5040 of LNAI, pages 447-456. Springer-Verlag, July 2008. http://heikohamann.de/pub/hamannSab08.pdf 
Heiko Hamann, Bernd Meyer, Thomas Schmickl, and Karl Crailsheim. A model of symmetry breaking in collective decision-making. In S. Doncieux, B. Girard, A. Guillot, J. Hallam, J.A. Meyer, and J.B. Mouret, editors, From Animals to Animats 11, volume 6226 of LNAI, pages 639-648. Springer-Verlag, 2010. http://heikohamann.de/pub/hamannSAB10.pdf

Heiko Hamann, Thomas Schmickl, Heinz Wörn, and Karl Crailsheim. Analysis of emergent symmetry breaking in collective decision making. Neural Computing \&3 Applications, 21(2):207-218, March 2012. http://heikohamann.de/pub/hamannNeuralCompApp10.pdf

Kristina Lerman and Aaram Galstyan. Mathematical model of foraging in a group of robots: Effect of interference. Autonomous Robots, 13:127-141, 2002.

Michael James Lighthill and Gerald B. Whitham. On kinematic waves. II. A theory of traffic flow on long crowded roads. Proceedings of the Royal Society of London, A229(1178):317-345, May 1955.

Hani S. Mahmassani, Jing Dong, Jiwon Kim, Roger B. Chen, and Byungkyu Park. Incorporating weather impacts in traffic estimation and prediction systems. Technical Report FHWA-JPO-09-065, U.S. Department of Transportation, September 2009.

Dejan Milutinovic and Pedro Lima. Cells and Robots: Modeling and Control of Large-Size Agent Populations. Springer-Verlag, 2007.

Octavio Miramontes. Order-disorder transitions in the behavior of ant societies. Complexity, 1(1):56-60, 1995.

Francesco Mondada, Michael Bonani, André Guignard, Stéphane Magnenat, Christian Studer, and Dario Floreano. Superlinear physical performances in a SWARM-BOT. In Mathieu S. Capcarrere, editor, Proc. of the 8th European Conference on Artificial Life (ECAL), volume 3630 of Lecture Notes in Computer Science, pages 282-291. Springer-Verlag, 2005. ISBN 9783540288480.

Julien Nembrini, Alan F. T. Winfield, and Chris Melhuish. Minimalist coherent swarming of wireless networked autonomous mobile robots. In Bridget Hallam, Dario Floreano, John Hallam, Gillian Hayes, and Jean-Arcady Meyer, editors, Proceedings of the seventh international conference on simulation of adaptive behavior on From animals to animats, pages 373-382, Cambridge, MA, USA, 2002. MIT Press.

Akira Okubo. Dynamical aspects of animal grouping: Swarms, schools, flocks, and herds. Advances in Biophysics, 22:1-94, 1986.

Akira Okubo and Simon A. Levin. Diffusion and Ecological Problems: Modern Perspectives. Springer-Verlag, Berlin, Germany, 2001. 
Amanda Prorok, Nikolaus Correll, and Alcherio Martinoli. Multi-level spatial models for swarm-robotic systems. The International Journal of Robotics Research, 30(5):574-589, 2011.

Thomas Schmickl and Heiko Hamann. BEECLUST: A swarm algorithm derived from honeybees. In Yang Xiao, editor, Bio-inspired Computing and Communication Networks. CRC Press, March 2011.

Steven H. Strogatz. Exploring complex networks. Nature, 410(6825):268-276, 2001.

http://www .nature.com/nature/journal/v410/n6825/abs/410268a0.html

Tamás Vicsek and Anna Zafeiris. Collective motion. arXiv:1010.5017v2, 2012. http://arxiv.org/pdf/1010.5017v2

Christian A. Yates, Radek Erban, Carlos Escudero, Iain D. Couzin, Jerome Buhl, Ioannis G. Kevrekidis, Philip K. Maini, and David J. T. Sumpter. Inherent noise can facilitate coherence in collective swarm motion. Proceedings of the National Academy of Sciences, 106(14):5464-5469, 2009. doi: 10.1073/ pnas.0811195106. http://www.pnas .org/content/106/14/5464.abstract 